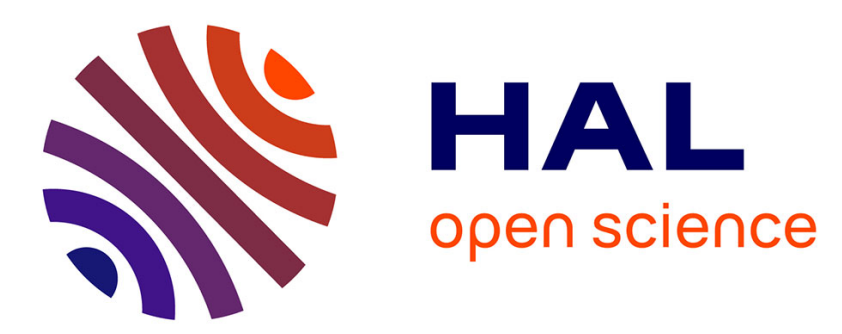

\title{
Permanences et réformes dans le secteur du logement en Lettonie depuis 1991
}

Eric Le Bourhis

\section{To cite this version:}

Eric Le Bourhis. Permanences et réformes dans le secteur du logement en Lettonie depuis 1991. Revue d'Etudes Comparatives Est-Ouest, 2010, Les aspects sociaux de la transition, 41 (2), pp.171 - 194. 10.4074/S033805991000207X . halshs-01379098

\section{HAL Id: halshs-01379098 \\ https://shs.hal.science/halshs-01379098}

Submitted on 11 Oct 2016

HAL is a multi-disciplinary open access archive for the deposit and dissemination of scientific research documents, whether they are published or not. The documents may come from teaching and research institutions in France or abroad, or from public or private research centers.
L'archive ouverte pluridisciplinaire HAL, est destinée au dépôt et à la diffusion de documents scientifiques de niveau recherche, publiés ou non, émanant des établissements d'enseignement et de recherche français ou étrangers, des laboratoires publics ou privés. 


\title{
Permanences et réformes DANS LE SECTEUR DU LOGEMENT EN LETTONIE DEPUIS 1991
}

\author{
ERIC LE BOURHIS
}

Ingénieur en Génie urbain, co-rédacteur en chef de Regard sur l'Est (eric.le-bourhis@regard-est.com)

Résumé : En Lettonie, la transition dans le secteur du logement est animée par le désengagement progressif de l'État depuis le milieu des années 1990. La mise en œuvre des politiques réformatrices du logement a rencontré des obstacles qui freinent aujourd'hui la rénovation du parc et a donné naissance à un système du logement bien particulier, se distinguant à maints égards de celui des pays voisins. L'auteur propose ici, en se fondant sur l'expérience de sept municipalités et du ministère de l'Économie, une étude du système letton du logement en transition. Celle-ci comprend une analyse de l'évolution des politiques menées et de leurs échecs, notamment un bilan de la privatisation, et une présentation de la conception du logement social ainsi que du système de gestion et d'entretien de l'habitat.

Mots-cLés : politiques du logement, logement social, mode d'occupation, transition, Lettonie, pays baltes. 


\section{INTRODUCTION}

Le système du logement en Lettonie est souvent qualifié de déficient : le retard de la rénovation de l'habitat et la difficulté à trouver un logement dans un contexte de pauvreté prononcée révèlent une crise du logement que les acteurs publics peinent à résoudre. Les obstacles à la restructuration globale du secteur, entreprise par le gouvernement et les municipalités depuis 1991, sont en particulier mis en cause. Comment ces acteurs perçoivent-ils la crise du logement? Quels sont les mécanismes de l'évolution de la politique du logement en Lettonie ? Nous appuyant sur l'expérience et le point de vue de sept municipalités et du ministère de l'Économie, nous proposons ici une étude du système letton du logement en transition où l'exposé des politiques menées depuis 1991 est suivi de leur évaluation et de l'analyse de leurs échecs.

\section{LA POLITIQUe DU LOGEMENT}

En Lettonie, la politique du logement est conduite au nom de la promotion du choix des personnes, synonyme du droit à devenir propriétaire : la clef de toutes les réformes du secteur est la privatisation du parc public, amorcée en Union soviétique en 1989, confirmée et accélérée après 1991. Ce contexte marque profondément l'évolution du cadre légal et des instruments que nous évoquerons ici.

\subsection{CAdre LÉgal et outils mis en Place entre 1991 et 2009}

Le premier acte législatif relatif à l'habitat, une fois l'indépendance recouvrée, concerne la dénationalisation, c'est-à-dire la restitution à leurs anciens propriétaires des immeubles nationalisés après le 17 juin 19401. $\mathrm{Au}$ même moment, le statut de propriétaire des habitants des kolkhozes et des membres des coopératives de construction ${ }^{2}$ est confirmé par la loi. Une large décentralisation vers les communes accompagne ces textes: la privatisation du parc public (c'est-à-dire faisant partie du patrimoine immobilier de l'État, de ses entreprises ou des muncicipalités), votée en 1995, est pour l'essentiel menée à bien par les communes.

1. Date marquant le début de l'occupation de la Lettonie par l'Union soviétique. Cette restitution a été massive, en particulier au bénéfice de familles résidant à l'étranger.

2. La propriété privée des coopératives est reconnue en URSS en 1988. 


\section{Cadre légal de la privatisation}

Loi du 21 juin 1991 «Sur la privatisation des entreprises agricoles et des kolkhozes de pêcheurs ».

Lois du 30 octobre 1991 «Sur la dénationalisation de la propriété immobilière en République de Lettonie » et «Sur la restitution de la propriété immobilière aux propriétaires légaux ».

Loi du 4 décembre 1991 «Sur la privatisation des coopératives de logement ».

Décret du Conseil des ministres n 307 du 4 août 1992 «Sur les sociétés coopératives de propriétaires de logement ».

Loi du 21 juin 1995 « Sur la privatisation du parc de logements de l'État et des municipalités $»^{3}$.

Loi du 28 septembre 1995 «Sur la propriété du logement».

À partir de la fin des années 1990, c'est l'aide au logement qui connaît une restructuration d'envergure. Avec les modifications apportées aux premiers textes, qui avaient été votés en 1993, le logement social est progressivement limité au secteur public, tandis que les critères d'accès à ce parc sont restreints. Les municipalités sont par ailleurs obligées de développer une offre locative accessible pour répondre à la demande.

Cadre légal de la conception de l'aide au logement

Loi du 16 février 1993 «Sur la location de pièces habitables ${ }^{4}$ (modifiée le 5 juillet 2001).

Loi du 11 mai 1993 «Sur l'aide de l'État et des municipalités dans la résolution de la question du logement».

Loi du 12 juin 1997 « Sur les appartements sociaux et les maisons sociales ».

Loi du 6 décembre 2001 «Sur l'aide à la résolution de la question du logement $»^{5}$.

La Conception de la politique du logement est adoptée par le Conseil des ministres pour organiser ces réformes et créer les instruments nécessaires à leur application en 1996 (Conseil des ministres, 1996). Elle affirme tout d'abord la volonté de défendre les propriétaires et les locataires, d'aider les pauvres à se loger, de rénover le parc existant, de liquider les foyers de travailleurs et les appartements communautaires et de rompre avec le passé en réorganisant totalement l'économie du secteur : il faut " permettre à la population de choisir son domicile en fonction de ses capacités matérielles ». L'État s'attache donc à concevoir des mécanismes de crédit pour la construction, l'acquisition et la rénovation ${ }^{6}$ - la part du secteur

3. Littéralement «Loi de privatisation des maisons habitables de l'État et des municipalités » (Par valsts un pašvaldību dzīvojamo māju privatizāciju). Nous la désignerons par l'expression de «loi de privatisation».

4. Loi que nous désignerons par les termes : « loi sur la location».

5. Loi que nous désignerons par les termes : « loi sur l'aide au logement».

6. Les bases de ces mécanismes sont validées en 1997. Le premier programme de crédits à long terme pour la construction, la reconstruction et la modernisation de l'habitat est, à proprement parler, mis en place en 2000 et il est suivi d'un second en 2002. 
public dans le parc est réduite de moitié entre 1995 et 2000 - mais le succès reste mitigé jusqu'en 2002 pour la construction (voir Graphique 1).

Une Agence du logement est alors créée auprès du ministère de la Protection de l'environnement et du développement régional pour coordonner La politique du logement en Lettonie (Ministère de la Protection de l'environnement, 2002). Les objectifs qu'elle fixe soulignent l'importance de la relance de la construction et les enjeux de la question du financement. En effet, la seconde phase du programme de financement parvient à compenser les erreurs de la première : pour aider les jeunes familles, un système de garanties permet de réduire l'apport initial, fixé en moyenne par les banques à $10 \%$ du prix de vente (Loseva, 2004).

GRAPHIQUe 1

Surfaces de logement construites en Lettonie entre 1980 et 2008

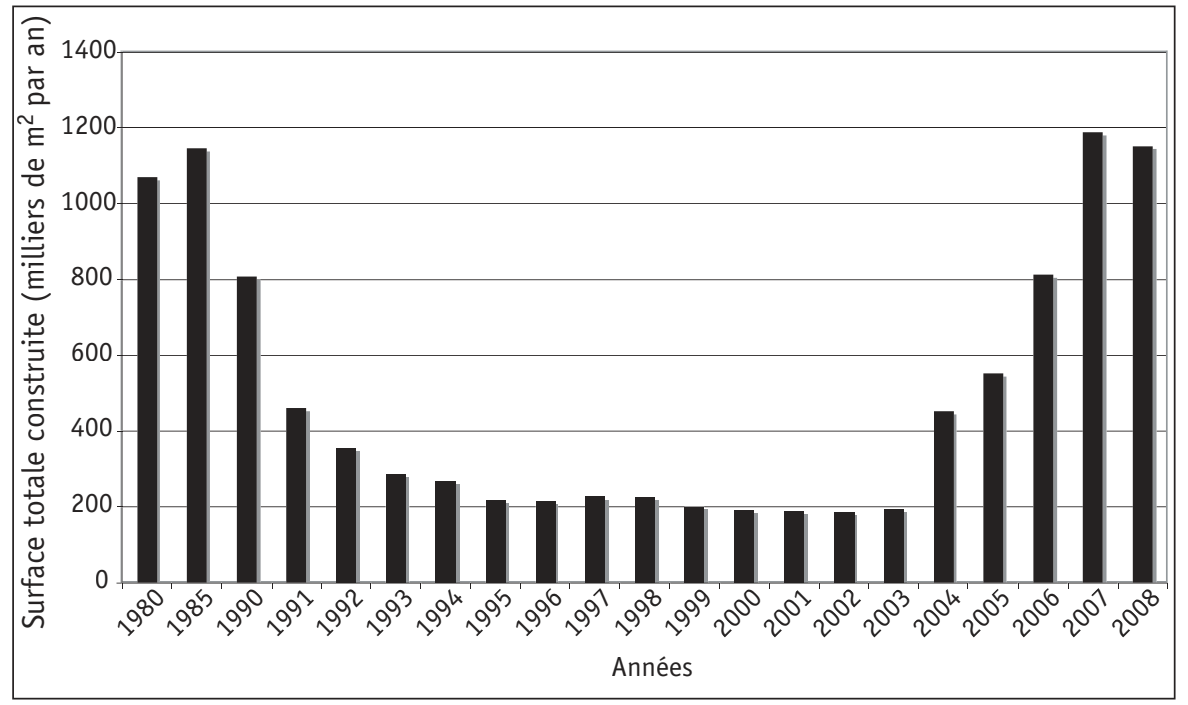

Note: L'État ne finance plus la construction de logement depuis 1995 (Loseva, 2004). Le volume de construction en 2009 est estimé aux deux tiers de celui de 2008.

Source : Latvijas Statistika (Office central de la statistique de la république de Lettonie), mai 2009; www.csb.gov.lv

Enfin, depuis le milieu des années 2000, l'agence du Logement ${ }^{7}$ (BEMA) concentre ses efforts sur la rénovation du parc privé. Depuis 2007, une modification de la loi sur l'aide au logement permet aux municipalités de distribuer des aides financières aux propriétaires pour la rénovation de leur immeuble, et instaure un programme, financé par l'État, d'améliora-

7. Passée sous la responsabilité du ministère de l'Économie en 2006 et renommée agence de l'État pour la Construction, les performances énergétiques et le logement (Būvniecības, enerǵêtikas un mājokḷ valsts aǵentūra).

VOLUME 41, JUIN 2010 
tion des performances énergétiques de l'habitat (2008-2010) ${ }^{8}$, appuyé par des fonds de cohésion européens depuis 2009.

\subsection{LA PRIVATISATION DU PARC PUBLIC}

Revenons à présent sur le processus de privatisation qui est au cœur des réformes du logement. D'une manière générale, il consiste à transférer le parc public et son entretien au secteur privé. Il conduit à limiter le rôle de l'État et à instaurer un marché de l'immobilier. La privatisation des logements publics a été observée pour la première fois à grande échelle dans l'Angleterre thatchérienne des années 1980 puis dans toute l'Europe de l'Est (Ghékière, 2007a, p. 95). En Lettonie, le terme privatizācija désigne, à proprement parler, l'accession à la propriété des locataires du parc public (contre paiement) et la mise en vente des appartements vacants. Le phénomène touche particulièrement les constructions de l'époque soviétique situées en milieu urbain. D'une part, en effet, l'habitat rural (soit environ un tiers du parc de logements total en surface) est déjà pour moitié propriété privée en 1990. D'autre part, le parc rural public est en grande partie restitué aux anciens propriétaires (80 000 appartements) et, lorsqu'il appartient à un kolkhoze (45 000 appartements), «privatisé » selon une modalité spécifique fixée par une loi adoptée dès 1991 (BEMA, 2009). Le processus de privatisation, voté en 1995, est principalement accompli avant 2001, avec un pic de 140000 transactions en 1999. Sur un parc total de près d'un million de logements, il a concerné 430000 appartements jusqu'en 2008, soit les trois quarts du parc public initial. Aujourd'hui, l'État et les municipalités ne possèdent plus que $12 \%$ du parc d'habitation (en surface), abritant selon nos estimations environ $15 \%$ des ménages (Tableau 1).

Tableau 1

Propriété du parc de logements en 2009 (\% du parc total en surface)

\begin{tabular}{|l|l|l|l|l|}
\cline { 2 - 5 } \multicolumn{1}{c|}{} & 1991 & 1995 & 2000 & 2009 \\
\hline Parc public & 69 & 50 & 24 & 12 \\
\hline Parc privé & 31 & 50 & 76 & 88 \\
\hline
\end{tabular}

Note : D'après nos évaluations, en 2009, les propriétés de l'État représentent $1 / 6^{\mathrm{e}}$ du parc public et la location privée $1 / 12^{\mathrm{e}}$ du parc privé.

Source: Latvijas Statistika.

La loi de privatisation précise le déroulement du processus : une commission municipale de Privatisation choisit les immeubles à privatiser, estime la valeur de chaque bien (appartement doté de la part correspondante des parties communes et de la parcelle) puis fait une proposition de transaction au locataire. Si celui-ci y répond favorablement, la commission

8. Amendements à la loi sur l'aide au logement, tous deux adoptés en 2007 (article 27.2). 
prend le cas échéant une décision de privatisation qui laisse trois mois au locataire pour acheter son appartement. Le paiement s'effectue avant tout par « certificats » (comme en Lituanie, en Estonie ainsi qu'en Russie avant la loi de privatisation gratuite de 1992), l'écart entre la valeur estimée de l'appartement et le montant des certificats devant être complété par un paiement en lats LVL pouvant être échelonné sur dix ans. Ces certificats, compromis entre une réflexion gorbatchévienne menée depuis 1989 (Amestoy, 2001) et des recherches entreprises à l'Ouest pour la création d'une économie de marché dans les anciennes républiques soviétiques (Vasiljevs, 2005), seront le support de la privatisation de toute l'économie après 1992. Ils sont transmissibles par héritage et peuvent être mis en vente sur le marché. En moyenne, une famille constituée de deux adultes de 40 ans et d'un enfant de 15 ans pouvait acquérir, grâce aux certificats, un appartement de $70 \mathrm{~m}^{2}$ (Tsenkova, 2004).

La loi de 1995 « Sur les certificats de privatisation » indique qu'ils seront distribués par l'État aux citoyens et aux habitants de la Lettonie selon le nombre d'années passées dans le pays (un par année avant 1992), aux victimes de la répression politique mais aussi aux anciens propriétaires qui, pour une raison particulière, ne peuvent bénéficier de la dénationalisation. Ces principes, qui désavantagent les jeunes ménages, traduisent un refus de distribution égalitaire qui aurait pu « profiter » à certains migrants soviétiques. Au-delà d'une date limite, repoussée à plusieurs reprises - le 31 août 2006 finalement -, les certificats ne sont plus utilisables. La vente reste possible mais le paiement doit s'effectuer en lats LVL. $95 \%$ des transactions conclues à ce jour l'ont été avant cette date.

\subsection{LA REDÉFINITION DE L'AIDE AU LOGEMENT}

Premier aspect généralement étudié de l'action publique dans ce domaine, l'aide au logement prend aujourd'hui en Lettonie la forme d'une location ou d'une aide financière accordée au cas par cas par les municipalités, en priorité aux personnes pauvres, âgées, invalides ou mineures ainsi qu'aux anciens déportés et aux personnes qui ont été expulsées de leur logement ; l'État et l'Union européenne financent en outre une partie des travaux de rénovation relatifs à l'amélioration des performances énergétiques de l'habitat. La loi sur l'aide au logement suit une logique qui sera celle adoptée par l'Union européenne, à laquelle le pays adhère en 2004 (Andria, 2007). Elle s'appuie sur la décentralisation, prend en compte le développement durable et le vieillissement de la population et, si elle n'instaure pas de véritable droit au logement, elle témoigne d'une volonté de fournir à chacun un domicile acceptable.

Dans la pratique, comme nous avons pu le constater, les communes privilégient un type d'aide. Dans les villes nouvelles (Aizkraukle fondée en 
1960 à proximité d'une nouvelle centrale hydroélectrique) ou reconstruites après la guerre (Jelgava, Valmiera), il n'existe pas de parc dénationalisé et peu ou prou de parc locatif privé : l'aide y est consacrée aux propriétaires (pour le chauffage notamment) ${ }^{9}$. En revanche, les municipalités des villes dotées d'un parc ancien (Riga, Cēsis) aident en priorité les locataires. Dans les discours de leurs représentants, c'est en effet l'expulsion de ces derniers qui est décrite comme la principale difficulté. Elle est autorisée après trois mois d'impayés et en cas de rénovation ou de reprise du logement par le propriétaire. Parce que les immeubles dénationalisés représentent une part très majoritaire du parc locatif privé - 87 \% en 2004) (Ščucka, 2004) -, ces municipalités disent orienter prioritairement leur action vers les locataires ou anciens locataires de ces immeubles (aide au paiement du loyer ou des charges, proposition de relogement). Cette tendance s'explique entre autres par la conviction que ces personnes, à qui l'État n'a pas offert la possibilité de privatiser leur appartement, ont été lésées. À Riga, en 2009, ces familles habitent ainsi $80 \%$ des logements municipaux ${ }^{10}$.

L'évolution du logement social en Lettonie depuis 1991 s'inscrit dans les tendances constatées par Laurent Ghékière dans les pays en transition. «Après une période de transition visant à rétablir un véritable marché du logement et de son financement, à privatiser le parc locatif public et face aux difficultés croissantes d'accès au logement, les nouveaux États membres vont progressivement réorganiser le secteur du logement social, notamment pour développer une offre locative accessible » (Ghékière, 2007a, pp. 94-95). Néanmoins, en Lettonie, à partir de la fin des années 1990, le développement d'une offre locative accompagne une redéfinition globale de la conception du logement social - redéfinition de sa forme et des catégories administratives qui en relèvent.

Prenons la définition du logement social qui est donnée par L. Ghékière : « réponse des pouvoirs publics à la défaillance structurelle du marché du logement à satisfaire l'ensemble des besoins en logement et à garantir à tous l'accès à un logement décent et accessible financièrement » (Ghékière, 2007a, p. 90). Selon cette définition, en Lettonie durant les années 1990, le logement social comprend toujours une importante offre locative publique (principalement en régie municipale) puisque, en 2000, la privatisation n'est effectuée qu'aux deux tiers, mais aussi un secteur privé qui permet de loger des personnes pauvres grâce aux plafonds de location et aux interdictions d'expulsion. Cela a pu laisser penser que l'État, comme en Russie ou en République tchèque, offrait une certaine alternative au marché libre (Tsenkova, 2004).

9. Entretien avec I. Žurzdina, assistante sociale de la ville d'Aizkraukle, 15 avril 2009. 10. Entretien avec A. Stabina, chef du bureau du Logement du Département communal de la ville de Riga, 14 juillet 2009. 
Dans les années 2000 cependant, tandis que l'achèvement de la privatisation oblige les municipalités à constituer un nouveau parc public répondant à la demande (10 000 personnes sur liste d'attente à Riga en 2003), le rôle du logement social est redéfini. En principe, celui-ci est désormais exclusivement réservé aux ménages jugés les plus vulnérables. La prolongation du délai d'utilisation des certificats conduit à la liquidation tardive du parc public (cf. Tableau 1) ; les critères d'accès sont restreints par la loi sur l'aide au logement de 2001 : la priorité est dès lors strictement accordée à des groupes cibles (personnes âgées, enfants, invalides, déportés, anciens détenus) à la condition que ceux-ci aient un revenu inférieur à un certain plafond $^{11}$, ainsi qu'aux personnes ayant été expulsées de leur logement. Parallèlement, les pauvres sont exclus du parc privé ${ }^{12}$ par suppression progressive des plafonds de location (entre 2002 et 2004), augmentation du montant contrôlé des charges ${ }^{13}$ et arrivée à échéance des interdictions d'expulsion dans le parc dénationalisé (7 ans à compter de la restitution). Il semble que la Lettonie soit passée en quelques années d'une conception généraliste à une conception résiduell $\mathrm{e}^{14} \mathrm{du}$ logement social.

TABLEAU 2

Le logement locatif municipal en Lettonie en 2008 (\% du parc total en surface)

\begin{tabular}{|l|c|c|c|c|c|c|}
\hline & Lettonie & Riga & Liepāja & Jelgava & Ventspils & Aizkraukle \\
\hline Population (milliers) & 2261 & 713 & 85 & 65 & 43 & 9 \\
\hline Chômage (mars 2009) & $11 \%$ & $8 \%$ & $13 \%$ & $10 \%$ & $10 \%$ & $10 \%$ \\
\hline Logement municipal & $10 \%$ & $3 \%$ & $19 \%$ & $1 \%$ & $14 \%$ & $8 \%$ \\
\hline $\begin{array}{l}Y \\
\text { Y compris appartements } \\
\text { sociaux }\end{array}$ & $0,4 \%$ & $0,3 \%$ & $1 \%$ & $0,4 \%$ & $0,3 \%$ & $0,5 \%$ \\
\hline
\end{tabular}

Note : Ce tableau ne prend pas en compte le parc de logement qui est propriété de l'État. Les taux de chômage sont donnés à titre indicatif pour permettre la comparaison ; le taux national atteint quant à lui 20 \% à la fin de l'année 2009.

Sources: Latvijas statistika; Nodarbinātības valsts aǵgentūra (Agence nationale pour l'emploi); données recueillies auprès des municipalités.

Précisons, par ailleurs, que la loi lettone ne qualifie de «sociale» qu'une part restreinte du parc municipal qu'elle désigne par le terme d'appartement social (sociālais dzìvoklis) depuis la loi de 1997. Il s'agit d'un logement

11. Fixé par chaque commune.

12. Malgré quelques projets récents mais non significatifs de logements conventionnés à destination de personnes défavorisées dans le parc privé à Liepāja ou Tukums.

13. Amendements adoptées en 2001 à la loi sur la location (règle complémentaire n ${ }^{\circ} 4$ et article 11)

14. C'est-à-dire dont « les missions sont strictement définies en référence au logement des personnes défavorisées et à des groupes sociaux clairement identifiés en raison de leur exclusion effective ou potentielle du marché du logement. » (Ghékière, 2007b, pp. 32-33) Ceci par opposition à une conception généraliste, plus diversifiée et strictement liée à l'insuffisance de revenus. 
locatif municipal très social dont le loyer est réduit de deux tiers et dont la part dans le parc est inférieure à $0,5 \%$ en surface au niveau national ${ }^{15}$ (Tableau 2). Il nous semble important de souligner qu'une définition très restrictive de l'habitat social satisfait le droit européen de la concurrence : « la location de logements aux ménages autres que socialement défavorisés ne peut être considérée comme un Service d'Intérêt Général » (Ghékière, 2007b, p. 40). Reconnu comme tel depuis 2007, ce type de logement peut donc prétendre à une subvention européenne.

\section{2. ÉVALUATION DU SYSTÈME DU LOGEMENT EN TRANSITION}

L'interprétation des chiffres et de l'évolution de l'action publique précédemment exposés demande une évaluation de la transition dans le secteur du logement que nous proposons d'esquisser ici sur la base des éléments transmis par les acteurs institutionnels rencontrés.

\subsection{LE BILAN DE LA PRIVATISATION}

Est-il possible de faire un bilan de la privatisation de l'habitat? Avec trois quarts des logements habités par leur propriétaire, la Lettonie est devenue un pays de propriétaires occupants. Pour l'agence de l'État pour la Construction, les performances énergétiques et le logement (BEMA), les objectifs de la privatisation sont dès lors atteints : création d'un marché de l'immobilier et normalisation du secteur. En effet, dès les années 1990, malgré le maintien de la pratique soviétique de l'échange d'appartement, le marché de la vente s'est développé grâce au commerce des certificats et à la pratique de l'hypothèque et s'est imposé comme le principal mode d'accession. À la fin des années 1990, les prix de vente se sont envolés du fait de l'accès au crédit et de la croissance économique. Toutefois, deux interrogations demeurent : que signifie le succès relatif de la privatisation et peut-on considérer qu'elle est réellement achevée ?

En Lettonie, la privatisation est tardive : la loi est votée en 1995 et le processus ne commence qu'en 1996 alors qu'au même moment, il est pratiquement achevé en Lituanie et en Estonie (lois de 1991 et 1993). De plus, si l'on mesure son succès au nombre de transactions réalisées avant 2006 parmi les appartements proposés, on constate qu'elle est très inégalement achevée. Pour une moyenne nationale de $86 \%$, on observe que la privatisation a été plus massive à Riga $(96 \%)$ et dans sa région (88 \%) qu'en province, en particulier en Courlande ( $72 \%$, mais $34 \%$ dans le rajons de Ventspils). En 2008, dans presque $20 \%$ des immeubles offerts à la privati-

15. Ce faible taux explique pourquoi, dans les études comparatives, la Lettonie est souvent classée à tort parmi les pays européens au plus faible taux de logement social (arrondi à 1 \%). 
sation, moins de la moitié des appartements ont eux-mêmes été privatisés (BEMA, 2009).

Par conséquent le secteur public, qui possédait $50 \%$ des surfaces habitables en 1995, en détient encore $12 \%$ en 2008 (Tableau 1), taux parmi les plus élevés de l'Europe de l'Est après la Russie et la République tchèque, contre moins de $3 \%$ en Estonie et en Lituanie (Eesti Statistika, 2009; Statistikos departamentas, 2009). De nombreux facteurs ont retardé ou empêché le processus : l'attrait relatif du statut de locataire public, l'accès difficile au crédit, le mauvais état du parc, la difficulté d'utilisation des certificats, le maintien dans le patrimoine de l'État de certains immeubles ou encore le refus de certaines communes de privatiser en bloc. On trouve ainsi les plus grands parcs publics dans les villes dont les municipalités ont opposé un tel refus et où le succès de la privatisation (Ventspils $75 \%$, Liepāja $84 \%$ ) a été moindre ${ }^{16}$ (Tableau 2) tandis qu'un tel parc est inexistant dans de nombreuses zones rurales et inférieur à $5 \%$ dans la plupart des villes. Le parc public comprend des logements de fonction (très peu nombreux), des logements attribués dans le cadre de la loi sur l'aide au logement de 2001 mais également des appartements non privatisés. En effet, les locataires du parc public qui occupaient leur appartement depuis l'époque soviétique et ne s'en sont pas portés acquéreur, soit parce qu'ils ne l'ont pas souhaité ou ne l'ont pas pu, soit parce qu'on ne le leur a pas proposé, jouissent aujourd'hui, conformément à la loi, d'un contrat de location sans limitation de durée et transmissible à un membre de leur famille. Locataires du parc public (municipal ou d'État), ils bénéficient d'un loyer avantageux bien qu'ils ne répondent pas aux critères d'attribution du logement social ${ }^{17}$. La loi de 2002 «Sur l'aliénation des propriétés de l'État et des municipalités » permet aux municipalités de vendre ces appartements mais n'est pas appliquée en raison des protestations de plusieurs municipalités, dont Riga (Kronberga, 2005).

Le succès de la privatisation peut également se mesurer à l'accomplissement de ses deux dernières étapes que sont l'enregistrement au cadastre exigé par l'article 48 et la constitution d'une «société de copropriétaires » (kopapsaimniekošanas sabiedrība) exigée par l'article $50^{18}$. Ce dernier article, précisé par les articles 8 et 27 de la loi «Sur la propriété du logement » de 1995 ainsi que par l'article 1068 du Code civil, dispose que, pour

16. Entretien avec D. Ošenieks adjoint au maire de Ventspils, 20 avril 2009. Entretien avec M. Tìdens, directeur du bureau de la Propriété immobilière de la ville de Liepāja, 23 avril 2009.

17. Nous n'avons pas trouvé de statistique officielle à ce sujet. D'aucuns estiment la part des logements de ce type dans le parc total de Riga à $5 \%$, y compris ceux qui appartiennent à l'État.

18. L'article 2 de la loi de privatisation mentioinne également comme objectif «l'incitation à l'entretien de l'habitat».

VOLUME 41, JUIN 2010 
administrer et entretenir les parties communes, l'assemblée générale des copropriétaires doit déléguer son pouvoir de décision à un copropriétaire ou créer une société de copropriétaires, au statut de société privée, qui peut par ailleurs désigner un gestionnaire. De nombreux moyens incitatifs ont été mis en œuvre pour encourager les propriétaires à prendre en main la gestion de leur immeuble : séminaires, création d'une filière professionnelle des syndics, lancement d'une revue intitulée Pārvaldnieks (Gestionnaire), concentration des aides à la rénovation sur les copropriétés déjà formées... Cependant, la création de telles copropriétés a été très lente et souvent aucun mode de gestion n'a été choisi (dans 20000 maisons et immeubles). Seules 3500 copropriétés avaient été formées avant la fin de l'année $2008^{19}$ (BEMA, 2009, p. 4), soit moins du tiers des immeubles concernés, contre $90 \%$ en Estonie et en Lituanie déjà en 2006. Deux raisons sont avancées pour justifier cet écart. D'une part, le processus de privatisation a été plus rapide et plus simple dans ces deux pays (pas d'enregistrement au cadastre qui impose une clarification des droits fonciers, pas de privatisation de la totalité des parties communes en Lituanie) (E\&IC, 2006, pp. 21-29) ; d'autre part, des gestionnaires y ont été nommés par défaut tant que les copropriétés n'étaient pas formées, par le tribunal en Estonie, par les municipalités en Lituanie, moyen de pression efficace sur les nouveaux propriétaires ${ }^{20}$.

Globalement, en Lettonie, le processus de privatisation a conduit à une situation hybride où le parc public est un résidu complexe, multiforme et non désiré du processus et où les droits de propriété des appartements, des murs, des parties communes et de la parcelle du parc privatisé ne sont pas clarifiés.

\subsection{LA CRISE DU LOGEMENT}

La crise du logement revêt des aspects multiples et n'a pas éclaté en 1991. En effet, comme dans de nombreuses républiques soviétiques, en République socialiste soviétique de Lettonie, la surface habitable par personne était assez réduite $\left(19,9 \mathrm{~m}^{2} /\right.$ habitant en 1990 pour une moyenne de 16 en URSS) en raison de la densité de l'utilisation du parc (appartements communautaires, foyers) et des normes de surface des constructions neuves. En 1990, 22 \% des ménages étaient inscrits sur une liste d'attente de relogement (Estonie $10 \%$, Lituanie $17 \%$, Russie $25 \%$ ) car ils disposaient de moins de 5,2 $\mathrm{m}^{2}$ de surface habitable par personne (norme applicable en République socialiste soviétique de Lettonie) (Kahn, 1992, p. 57). Malgré les grands programmes de construction réalisés après 1960, l'obtention d'un logement est restée difficile jusqu'en 1990.

19. Dont seulement 412 sous la forme d'une société de copropriétaires.

20. Entretien avec I. Simanoviča, directrice du département du logement de l'agence de l'État pour la Construction, les performances énergétiques et le logement, 16 avril 2009. 
Après 1991, le contexte démographique change et la crise du logement prend un autre visage. Le pays perd $15 \%$ de sa population ${ }^{21}$, accroît la surface de son parc de logement de $1 \%$ entre 1991 et 2000 puis de $14 \%$ entre 2000 et 2008 (Figure 1). Celui-ci comporte environ un million de logements (dont $65 \%$ en ville) pour une population de 2,26 millions de personnes en 2009 ; la surface habitable est de $27 \mathrm{~m}^{2} /$ habitant, ce qui traduit une amélioration notable des conditions de vie. Toutefois le parc reste très normé : il compte surtout des studios et des appartements de deux pièces. Les grands appartements sont majoritairement situés à Riga et, souvent, encore communautaires (plus de 6000 en 1995 sur un total national de 8000 , dont 85 toujours loués par la municipalité en 2009) ${ }^{22}$.

Le fait que l'accès au logement dépende désormais du marché privé de l'immobilier, qui s'est emballé entre 1996 et 2007, provoque une nouvelle crise du logement. Les prix de vente quadruplent en moins de dix ans, jusqu'à 1500 euros le $\mathrm{m}^{2}$ en moyenne à Riga dans un immeuble construit à l'époque soviétique ${ }^{23}$. Si ce montant demeure plus faible qu'ailleurs en Europe, le prix du neuf y est parfois plus cher du fait des coûts de construction et des marges élevées des promoteurs (jusqu'à $50 \%$ ). Certes, la construction reprend à partir de 2003 mais elle concerne avant tout l'habitat urbain haut de gamme : c'est la région de Riga (la moitié de la population et trois quarts des emplois du secteur immobilier) qui bénéficie de $70 \%$ de ces nouvelles surfaces ${ }^{24}$. De surcroît, la concentration de la demande à Riga implique un assez grand nombre de sans-abris (estimé à 1000 en 2008), des migrations pendulaires quotidiennes de travail jusqu'à $100 \mathrm{~km}$ aller ${ }^{25}$ et la formation de ménages multigénérationnels lorsque les jeunes, en général locataires, retournent habiter chez leurs parents. Ailleurs, dans certaines zones de dépression et de chômage (dans la ville de Liepāja ou encore dans la région de Latgale), de nombreux appartements sont vides. Néanmoins, depuis l'éclatement de la bulle immobilière en 2007, les prix de vente ont chuté de $60 \%{ }^{26}$, incitant de nombreux propriétaires à louer plutôt qu'à vendre, même si les loyers sont également en baisse ${ }^{27}$. À Riga,

21. De 1991 à 2009, le solde naturel annuel moyen est de -5\% et le solde migratoire cumulé de 200000 départs.

22. Entretien avec A. Stabiņa, chef du bureau du Logement du Département communal de la ville de Riga, 14 juillet 2009.

23. Les immeubles des années 1970 et 1980 sont particulièrement prisés.

24. En 2002, la surface moyenne des nouveaux logements ont en moyenne $236,3 \mathrm{~m}^{2}$, soit deux fois plus qu'en Estonie ou en Lituanie (Tursons, 2004).

25. Entretien avec E. Zēbergs, secrétaire général, et L. Zībārte, présidente de la commission de Privatisation de la ville d'Aizkraukle, 15 avril 2009.

26. «Krīzes beigas prognozē péc gada» (La fin de la crise est prévue pour dans un an), Latvijas Vēstnesis, 16.01.

27. La location concerne $7 \%$ des surfaces (Estonie - 9\%, Lituanie - 2\%) et environ $10 \%$ des ménages. Ces estimations de l'auteur, faute de statistiques officielles, sont néanmoins incertaines du fait des fluctuations récentes et de la grande proportion de locations sans bail 
le prix des locations (qui avait triplé en dix ans) a été divisé par deux en un an et le nombre de contrats de location signés a fortement augmenté. La chute de plus de $10 \%$ des salaires en moyenne en un an et le chômage (20\% fin 2009, contre $5 \%$ fin 2007), l'interruption de la création d'une nouvelle offre locative à caractère social du fait de la réduction drastique du budget de l'État et des communes en 2009 relativisent la facilité de trouver un logement malgré la chute des prix.

C'est bien à la fin des années 1990 que s'est opérée une prise de conscience des difficultés évoquées précédemment, suivie d'une redéfinition de la politique sociale du logement. Le risque d'expulsion s'est en effet concrétisé lors de la crise de 1998 quand plus de 4000 expulsions sont prononcées par le tribunal, touchant $0,5 \%$ des ménages (Leckie, 2000). À la fin des années 1990, les premières échéances de maintien dans les murs des anciens locataires ont coïncidé avec la crise financière russe. Certes, l'appareil législatif letton prévoit des garanties contre l'expulsion (obligation au propriétaire ou à la mairie de reloger la personne, sept ans d'interdiction d'expulsion dans les immeubles dénationalisés) et l'attribution d'aides en cas d'expulsion. Néanmoins, le relogement n'est obligatoire que dans des cas très restreint ${ }^{28}$ et les villes disposent souvent d'un parc public insuffisant pour aller au-delà de leur devoir alors que les locataires n'ont à leur disposition que quelques institutions vers qui se tourner (mairie d'Ogre, association de locataires à Liepāja). En outre, l'augmentation, après 2002, des loyers et des charges a fragilisé une population, classée aujourd'hui par Eurostat comme ayant le plus haut risque de pauvreté de l'Union européenne (Eurostat, 2010). La part relative moyenne des dépenses de logement dans les revenus d'un ménage atteint $25 \%$ en 2007 (Estonie $21 \%$, Lituanie $14 \%$ ) (Czischke, 2007) voire davantage pour les retraités, ce qui conduit à un endettement rapide en cas d'hiver froid ${ }^{29}$ ou de crise économique.

Globalement, étant donné la nature chaotique du marché foncier, la rigidité de l'offre, la demande continue émanant des couches aisées de la population et des investisseurs étrangers et le difficile accès au crédit, il est impossible de loger toute la population à un prix et des conditions d'hygiène acceptables (Marana, 2004). En outre, l'inefficacité de l'entretien, l'inexistence de la maintenance préventive, à l'époque soviétique comme aujourd'hui, ainsi que l'absence d'investissement dans la rénovation sont responsables d'une dégradation très visible des façades et des parties communes du parc ${ }^{30}$.

(évaluée à $30 \%$ ).

28. Articles 28.2, 28.3, 28.4 et 36.1 de la loi sur la location.

29. Le chauffage représente parfois jusqu'à $50 \%$ des dépenses de logement.

30. Entretien avec I. Simanoviča, directrice du département du logement de l'agence de l'État pour la Construction, les performances énergétiques et le logement, 16 avril 2009. 


\subsection{QUELLE EXPÉRIENCE DE LA MIXITÉ SOCIALE?}

Dans ce contexte de crise du logement, le caractère pluriethnique de la Lettonie pose assez naturellement la question de l'exclusion et de la mixité. Si le résultat de nos recherches ne permet pas de tirer de conclusion précise à ce sujet, il nous semble néanmoins pouvoir remettre en cause certains lieux communs. Il faut tout d'abord souligner que, malgré l'idéologie égalitaire, la ségrégation spatiale existait dans les villes lettones comme dans les autres villes de l'URSS (Ruoppila, 2004). S'agissant de l'obtention d'un logement, certains immigrants soviétiques étaient favorisés et des quartiers d'élite étaient réservés aux apparatchiks (Mežaparks à Riga, station balnéaire de Jürmala). Globalement, divers groupes ethniques ou sociaux furent regroupés : les Lettons dès 1945 dans les appartements communautaires, les populations en difficulté dans des quartiers dépeuplés et stigmatisés (tel le faubourg de Maskavas [Moscou] à Riga, à majorité russophone), les migrants soviétiques pour partie dans les grands ensembles grâce aux programmes de construction et aux critères de relogement mis en œuvre à partir de $1960^{31}$. Les coopératives de construction de logements, une forme de propriété largement développée en Lettonie dans les années 1980, profitèrent quant à elles à une population relativement aisée, en particulier les familles de militaires.

La situation actuelle hérite de ce contexte mais si le marché libre a atténué la ségrégation ethnique entre Russes et Lettons, il conduit à une sélection de la population selon ses revenus et à la concentration de personnes défavorisées dans des zones paupérisées telles qu'on les voit apparaître à l'échelle de quartiers à majorité russophone à Riga (faubourg de Maskavas) et Liepāja (ancienne base militaire de Karosta). À moindre échelle, plusieurs communes reconnaissent que les immeubles non privatisés, qui comptent des logements municipaux en location (rue de Limbaži à Valmiera, rue de la Gare à Jelgava), concentrent encore la population délinquante et la communauté rom «pour un loyer généralement impayé $»^{32}$, les appartements sociaux étant plutôt attribués à des personnes seules âgées ou invalides.

Comme si elle érigeait en principe la phrase de Pierre Bourdieu, «Rien n'est plus intolérable que la proximité physique (vécue comme promiscuité) de gens socialement éloignés » (Bourdieu, 1993, p. 166), la Lettonie ne goûte pas aujourd'hui le romantisme de la mixité sociale. Celle-ci existe dans certains grands immeubles, mais c'est plutôt de mauvais gré qu'elle est acceptée, notamment par des ménages en voie d'ascension sociale visà-vis de voisins occupants d'un appartement municipal. Un certain consen-

31. C'est-à-dire, en Lettonie, après la purge politique de 1959.

32. Entretien avec J. Baiks, secrétaire général de la ville de Valmiera, 6 avril 2009. Entretien avec R. Stūrāne, directrice des Affaires sociales de la ville de Jelgava, 9 juillet 2009. 
sus semble même émerger aujourd'hui parmi les acteurs institutionnels (mairies de Riga, Jelgava, Valmiera, Cēsis) contre ce qui est parfois désigné comme un leurre soviétique. Ainsi, l'intégration par le logement des personnes défavorisées ne fait pas débat ${ }^{33}$. Comme y invite la loi de 1997, les appartements sociaux sont souvent regroupés dans des immeubles spécifiques dits «maisons sociales » (sociālās mājas) parfois constituées dans d'anciens foyers construits à l'époque soviétique (Aizkraukle, Jelgava). Riga sert d'exemple: les appartements sociaux représentent $10 \%$ du parc municipal et sont rassemblés pour un tiers dans treize maisons socia$\operatorname{les}^{34}$; dans le quartier défavorisé de Maskavas, des policiers et assistants sociaux sont spécialement affectés à ces immeubles. Comme l'explique I. Sietinsone, directrice des Affaires sociales de Cēsis, malgré les réticences de son service, le logement municipal a été regroupé dans cinq immeubles, dont quatre dans un même quartier au sud-ouest de la ville ${ }^{35}$.

\section{LES ÉCHECS DE LA RESTRUCTURATION DU LOGEMENT}

En Lettonie, comme ailleurs, l'efficacité, voire l'existence même, d'une politique du logement est mise en doute par la population. Plus original, en revanche, est le scepticisme exprimé par certains acteurs institutionnels quant au succès même de leur pratique. La rénovation du parc est au cœur du débat.

\subsection{LES DISCOURS SUR L'ÉCHEC DE LA POLITIQUE DU LOGEMENT}

Dans les discours recueillis auprès des acteurs institutionnels rencontrés, la taille des immeubles dits soviétiques, la passivité de la population et le manque de concurrence apparaissent comme les causes objectives des échecs de la politique du logement, en particulier en ce qui concerne la formation des copropriétés et le financement de la rénovation. Autrement dit, l'héritage soviétique, matériel et social - un parc construit à l'époque soviétique et les pratiques sociales afférentes -, freinerait la restructuration du secteur et son corollaire, la rénovation du bâti.

Nous pensons que ces différents éléments sont liés entre eux et au déroulement du processus de privatisation. Celui-ci, en effet, s'est accompagné de la mise en valeur de l'image du propriétaire. L'objectif de la privatisation est bien l'accession à la propriété ainsi que le rappelle la Conception de la politique du logement (1996). De plus, la notion de propriété est étroitement associée à la représentation de la maison individuelle (savrupmāja

\footnotetext{
33. Plusieurs ministères n'ont pas souhaité nous rencontrer pour des entretiens portant sur ce sujet.

34. Entretien avec A. Stabina, chef du bureau du Logement du Département communal de la ville de Riga, 14 juillet 2009 .

35. Entretien réalisé le 8 avril 2009.
} 
en letton, de savrup - isolé et māja - maison). Ce même document précise qu'un des deux « objectifs du changement des propriétés structurelles du parc de logements est l'augmentation de la proportion de maisons individuelles et de maisons de peu d'étages » (article 15), conformément au désir de la population - selon la Politique du logement en Lettonie, publiée en 2002, les deux tiers de la population interrogée par sondage souhaite acquérir une maison. L'image des maisons individuelles est ainsi largement présente dans les documents de communication adressés aux municipalité $^{36}$ et à la population et ce, même lorsqu'ils s'adressent spécifiquement à des copropriétaires : la photographie d'une maison individuelle est projetée et commentée durant le séminaire d'information des copropriétaires, organisé à Cēsis par la municipalité et l'office du logement CDzP le 7 avril 2009, auquel nous avons assisté. La presse non spécialisée vante, quant à elle, les mérites de l'habitat pavillonnaire périurbain ${ }^{37}$ présenté comme original et beaucoup plus propice à la vie familiale (Smilǵge, 2008). Dans ce cadre, les immeubles construits à l'époque soviétique, qui abritent néanmoins la moitié de la population du pays et constituent la perspective ultime de nombreux ménages en voie d'ascension sociale, sont perçus comme des anomalies et indirectement visés à ce titre. Ils seraient difficiles à privatiser du fait du grand nombre de propriétaires et se prêteraient mal à la formation de copropriétés ${ }^{38}$. Et ce topos a du succès dans une nation qui cherche ses racines dans la nature et l'habitat traditionnel : l'habitat individuel est parfois même associé à l'identité lettone par opposition à un colon soviétique, regardé comme porteur d'habitat collectif ${ }^{39}$.

Une autre cause est mise en avant : la passivité, c'est-à-dire le manque de volontarisme dans la formation de copropriétés et les projets de rénovation, voire l'absence totale de mobilisation de la population ${ }^{40}$, aliénée par cinquante ans d'occupation soviétique : «L'héritage soviétique est un réel problème. Les gens ne s'intéressent pas à ce qui se passe à l'extérieur de leur appartement. $»^{41}$ Le document d'évaluation des possibilités de revitalisation de la ville de Riga (E\&IC, 2006, p. 31) certifie lui aussi qu'il « est plus difficile d'impliquer les propriétaires d'appartements privatisés dans les processus de rénovation des immeubles » que de mener des pro-

36. La couverture de ce dernier document est illustrée par le dessin d'un lotissement pavillonnaire.

37. Les maisons individuelles représentent $28 \%$ des logements et les deux tiers des surfaces habitables du rajons de Riga (principale couronne de la périphérie).

38. Entretien avec G̣. Beikmanis, directeur de l'entreprise CDzP (Cēsis), 7 avril 2009.

39. Entretien avec R. Liepiňš, architecte, directeur de l'agence d'architecture Sudraba. Riga, 20 avril 2007.

40. Entretien avec G. Beikmanis, directeur de l'entreprise CDzP (Cēsis), 7 avril 2009. Entretien avec I. Krauze, directeur du département juridique de l'agence de l'État pour la Construction, les performances énergétiques et le logement, 16 avril 2009.

41. Entretien avec E. Zēbergs, secrétaire général, et L. Zībārte, présidente de la commission de Privatisation de la ville d'Aizkraukle, 15 avril 2009. 
jets de démolition-reconstruction. Le fait que les aides pour l'amélioration des performances énergétiques ne sont allouées qu'aux copropriétés des immeubles «soviétiques » nous semble à ce titre exemplaire : il incite à être responsables les individus précisément soupçonnés d'inertie.

Ce discours sur la passivité, qui mériterait une recherche approfondie, nous semble donc lié à l'image du propriétaire entreprenant, plébiscitée par la privatisation, mais aussi, et d'une manière plus générale, au contexte du désengagement de l'État et de l'établissement d'une «société soumise à une dynamique concurrentielle » telle qu'a pu la décrire Michel Foucault dans ses cours au Collège de France (Foucault, 2004, pp. 139-154). En effet, le manque de concurrence est également cité comme une cause du retard des réformes ${ }^{42}$ puisque, justement, la mise en place de mécanismes concurrentiels en est le fondement, y compris dans le secteur de l'aide au logement. Xavier Desjardins, qui propose d'observer les conceptions du logement social en Europe occidentale à l'aune d'une telle définition de la gouvernance néolibérale (Desjardins, 2008), suggère, en reprenant les termes de Jacques Donzelot, que l'action publique, la politique sociale incluse, «n'est plus pensée comme un palliatif aux insuffisances du marché » mais « comme un moyen de construire la société de la concurrence ». Dès lors, il ne s'agit plus de protéger les individus mais de les inciter à la compétitivité, de les amener à se laisser gouverner par leur propre intérêt, conception qui nous paraît proche des propos que nous avons recueillis. L. Ghékière fait lui aussi le lien entre le désengagement de l'État, le changement d'échelle de l'action publique (la décentralisation) et le ciblage des aides sociales (Ghékière, 2007a, p. 95). L'État reporte ses responsabilités sur un niveau administratif local et sur les individus eux-mêmes ; l'aide est distribuée au cas par cas et à la demande, des concours sont organisés pour la distribution des aides à la rénovation et seuls les individus jugés inaptes profitent de ce qu'on appelle la cohésion sociale.

Ces différents topoï nous semblent refléter avant tout une distance entre la population et les acteurs institutionnels. Pour expliquer les lenteurs de la formation des copropriétés et de la rénovation du parc, nous souhaitons mettre en relief deux points présents en filigrane dans les déclarations recueillies : les difficultés de la décentralisation et le retard dans la rationalisation du système d'entretien.

\subsection{UNE DÉCENTRALISATION DIFFICILE}

En Lettonie, la décentralisation est très avancée puisqu'elle s'effectue de l'État vers les communes sans intermédiaire, le secteur du logement étant, pour de nombreuses communes, un sujet de désaccord avec la poli-

42. Entretien avec I. Krauze, directeur du département juridique de l'agence de l'État pour la Construction, les performances énergétiques et le logement, 16 avril 2009. 
tique nationale menée. Dans les années 1990, l'État s'est tout d'abord défait de son rôle de bailleur de logements. Si la loi de 1993 «Sur l'aide de l'État et des municipalités dans la résolution de la question du logement " le confirme encore dans ce rôle, l'État cesse de construire après 1995. Ensuite, la loi sur l'aide au logement de 2001, qui s'y substitue, consacre la pleine responsabilisation des municipalités à cet égard. L'État poursuit alors le transfert, entamé dans les années 1980, de son patrimoine immobilier à ces dernières ${ }^{43}$ et leur confie des missions d'organisation et de conseil : les services d'aide sociale et les offices municipaux d'entretien $\mathrm{du}$ logement deviennent les principaux interlocuteurs des habitants en la matière. Contrairement à la dénationalisation et à la «privatisation » des kolkhozes, réalisées conjointement par les rajons (départements), la privatisation des logements est accomplie par les seules municipalités. En 2009, avec la réforme administrative du territoire (loi du 18 décembre 2008), les rajons sont supprimés, concentrant de fait l'exécution de la politique du logement dans les mains des seules municipalités (novads). La décentralisation s'opère néanmoins vers des communes élargies (19 000 habitants en moyenne) puisque cette réforme impose leur fusion (de plus de 500 à 118 au total $)^{44}$.

Si la décentralisation implique l'octroi d'une certaine liberté d'action aux municipalités ${ }^{45}$, celles-ci doivent pourtant appliquer une politique qui ne les convainc pas :parmi les municipalités rencontrées, plusieurs affichent des désaccords avec la législation. En effet, dans un contexte de désengagement financier de l'État et de liquidation du parc public, la décentralisation pèse lourdement sur l'action municipale. Le texte obligeant les municipalités à fournir un logement aux personnes les plus défavorisées (loi de 2001) ayant été adopté après la privatisation, il contraint de nombreuses communes à constituer un nouveau parc social, ce que certaines d'entre elles ne sont pas en mesure de faire.

En outre, nous avons discerné deux points de discorde entre l'État et certaines communes. D'une part, le transfert de propriétés immobilières du premier aux deuxièmes (80 immeubles concernés en 2008) est souvent présenté comme un problème. Plusieurs communes dénoncent par exemple la stigmatisation de ce parc étatique, mal entretenu et mal fréquenté, et négocient les conditions financières de son transfert qui suppose l'entrée

43. En Lettonie, en 1991, l'État possédait déjà moins de 15 \% du parc public, pourcentage maintenu jusqu'à nos jours.

44. Certaines zones rurales auparavant privées d'aide sociale peuvent dès lors bénéficier des services d'une ville proche.

45. Par exemple, la loi sur l'aide au logement impose une aide minimum, les communes étant libres d'élargir son champ d'application et son montant. Durant l'hiver 2008-2009, la ville d'Aizkraukle a versé une aide pour le paiement des charges à $35 \%$ des ménages et celle de Cēsis à $2 \%$. 
dans leur patrimoine d'immeubles en état de dégradation avancé : quartier de la rue Dzelzcelnieku pour la ville de Ventspils, quartier de la gare pour la ville d'Aizkraukle ${ }^{46}$. Par ailleurs, le débat sur l'ampleur que devait avoir la privatisation a été largement médiatisé en Lettonie, en particulier dans la ville de Ventspils. La municipalité, qui ne souhaitait pas privatiser à court terme les immeubles construits à l'époque soviétique ${ }^{47}$, a été forcée par l'État, après un bras de fer politique, médiatique et juridique, d'offrir à la privatisation, après 2006, la quasi-totalité de son parc de logement. Dans certains cas, ces difficultés du processus de privatisation ont conduit à un blocage du financement de la rénovation.

\subsection{LE SYSTÈME DE GESTION ET D'ENTRETIEN DE L'HABITAT}

Le marché de la gestion et de l'entretien est balbutiant : quelques groupes immobiliers s'imposent dans les constructions neuves tandis que les habitants des immeubles de petite taille s'occupent eux-mêmes de l'entretien de leur copropriété. Les offices du logement, entreprises publiques héritières des administrations soviétiques du logement (nampārvalde), réalisent toujours l'entretien et la gestion de la plus grande partie du parc $^{48}$. Une grande confusion règne d'ailleurs en letton entre les termes désignant l'entreprise chargée de la maintenance à proprement parler (apsaimniekotājs) et le gestionnaire (pārvaldnieks). En effet, par défaut, lorsque les propriétaires n'ont pas décidé du mode de gestion de leur immeuble, c'est cette entreprise publique qui remplit la fonction de syndic et fait payer à chaque propriétaire un prix forfaitaire de charges, souvent identique à celui que payent les locataires (en moyenne $0,30 \mathrm{LVL} / \mathrm{m}^{2} / \mathrm{mois}$, soit $0,42 €$ - une réforme est toutefois en cours depuis 2009), et se contente d'assurer un service minimum dicté par l'urgence. De tels montants ne couvrant pas les dépenses nécessaires, ces entreprises endettées souffrent d'une très mauvaise image auprès de la population comme en témoigne les propos recueillis à Aizkraukle : « Tu vois comme la cage d'escalier est sale. Je ne sais pas ce qu'ils font. On paye 0,35 lats par mois mais ils ne font rien. On n'a pas vraiment de contrat avec eux. Je ne sais pas trop ce qu'ils sont censés faire. On écrit. Mais les gens en ont marre d'écrire. $»^{49}$

Alors qu'à l'époque soviétique, les loyers étaient dérisoires et que les locataires n'avaient à régler que 20 \% des charges, leur hausse depuis 2002 s'accompagne d'un discours sur le «coût réel », volontiers relayé par les

46. Il s'agit dans ces deux cas d'immeubles gérés par les Chemins de fer nationaux.

47. Ce que la loi l'autorisait a priori à faire, le choix des immeubles à privatiser relevant de la commission municipale de privatisation.

48. Phénomène également constaté en Lituanie et en Russie. Ces offices entretiennent le parc public, le parc privé en cours de privatisation, mais également et surtout une grande partie du parc privatisé.

49. Propos recueillis à Aizkraukle dans un immeuble privatisé, rue Lacplesis le 15.04.2009. 
offices du logement dont les ressources ne permettent pas de mener à bien la rénovation. La loi précise que le montant des charges doit couvrir les travaux de rénovation ${ }^{50}$ tandis que les débats sur leur montant optimal remplissent les colonnes de la presse. Ce discours, qui tente à la fois de faire prendre conscience du vrai coût de la rénovation et de l'inefficacité des offices publics face à une concurrence privée naissante, a été décrit à l'échelle européenne par L. Ghékière (Ghékière, 2007a, p. 96) et n'aurait pas d'autre fin que le désengagement de l'action publique dans le secteur. Cette logique achoppe néanmoins sur la pérennité du monopole quasi absolu des offices du logement dans le secteur de la gestion et de l'entretien, tel qu'on peut l'observer à l'échelle de nombreuses communes : Aizkraukle, Cēsis, Daugavpils, Jelgava, Valmiera. À Cēsis, l'entreprise de gestion, $\mathrm{CDzP}$, a été privatisée, mais jouit du même monopole que les offices municipaux des autres villes. À Riga, quinze entreprises municipales sectorisées entretiennent les trois quarts du parc total. Dans un quartier que nous avons retenu pour notre étude de terrain, celui de Tornakalns (office du logement Zemgale, $2^{\mathrm{e}}$ secteur), $63 \%$ des immeubles privatisés sont gérés par l'entreprise publique, $32 \%$ ont formé des copropriétés et, en l'absence de concurrent, ont choisi pour syndic la même entreprise et $5 \%$ s'en sont séparés pour gérer eux-mêmes leur immeuble.

De l'avis de l'agence de l'État pour la Construction, les performances énergétiques et le logement (BEMA), le système letton échoue à toutes les évaluations de performance. Deux critères sont avancés : la formation de copropriétés responsables pour permettre la rénovation de l'habitat et la rationalisation du secteur de l'entretien. D'après un rapport commandé par la ville de Riga (E\&IC, 2006), en Estonie, d'une part, la privatisation, effectuée selon un cadre légal très similaire, aurait été plus prompte et plus complète et su désigner rapidement des gestionnaires, ce qui aurait facilité le versement à un fonds spécial (Kredex) des crédits octroyés pour la rénovation. D'autre part, les offices publics du logement auraient été privatisés dans les meileurs délais, prévenant la constitution de monopoles en ce domaine mais empêchant néanmoins les municipalités de conserver un certain contrôle de l'état sanitaire des immeubles privatisés. En Lituanie, inversement, la privatisation aurait été moins ambitieuse, comme nous l'avons déjà évoqué, et encore plus rapide, ce qui a permis à un certain nombre d'immeubles de profiter dès 1996 d'un programme de rénovation cofinancé par la Banque mondiale puis, depuis 2004, d'un programme national de rénovation des parties communes et d'amélioration des performances énergétiques. Surtout et alors que, comme en Lettonie, les offices publics du logement s'occupent encore de la gestion et de l'entretien de la plupart des immeubles, le secteur a été professionnalisé et leur activité est

50. Article 11 de la loi sur la location, repris par la Conception de la politique du logement de 1996 (Conseil des ministres, 1996).

VOLUME 41, JUIN 2010 
contrôlée par une inspection nationale de la Planification territoriale et de la construction du ministère de l'Environnement ${ }^{51}$.

En Lettonie, au contraire, la moitié des immeubles est, d'après la BEMA, mal gérée et mal entretenue d'autant que l'activité de ces offices n'est pas contrôlée lorsqu'elle concerne des immeubles privatisés. Avant la réduction drastique de ses effectifs en juillet 2009, dans un contexte de restructuration des services publics imposée par l'Union européenne et le Fonds monétaire international, la BEMA tenait à signaler à la fois l'incompétence des professionnels du secteur et le manque de confiance de la population dans les organismes de crédit et dans les syndics. Le retard dans la formation des copropriétés bloque, lui, les projets de rénovation qui nécessitent généralement l'obtention d'un crédit, ce qui, en l'absence de gestionnaire, est pratiquement impossible. Rares sont les municipalités à avoir vraiment soutenu ce type de projet - Ventspils (avant privatisation) ou Valmiera (prêts à taux zéro) ${ }^{52}$ (E\&IC, 2006) - qui n'est plus subventionné qu'au nom du développement durable. En Lettonie, la lenteur de la rationalisation du système de gestion et d'entretien de l'habitat est bien une pierre d'achoppement de la rénovation du parc.

\section{Conclusion}

La transition du système du logement en Lettonie présente des anomalies qui peuvent trouver leur explication dans les obstacles aux réformes. En particulier, le maintien, à l'encontre des désirs du législateur, d'un vaste parc locatif public ainsi que le monopole mal contrôlé des offices publics du logement sont parfois perçus comme des moyens d'adoucir les conséquences sociales de la transition, autrement dit de permettre à une population pauvre de conserver son domicile, pendant que certains déplorent la surprotection de la population, cantonnée dans des conditions de vies médiocres et prétendument économiques. À ce titre, le thème de la permanence est très profondément ancré dans les discours des acteurs publics du logement, en particulier aujourd'hui pour les projets de rénovation. Face à la déshérence du parc, géré de manière inefficace, un texte à valeur coercitive a été adopté pour la première fois en juin 2009. La loi sur la gestion de l'habitat tente en effet de définir le champ des responsabilités respectives des propriétaires et des gestionnaires qui seront, par défaut, nommés par la commune comme en Lituanie. Ce texte, s'il cherche avant tout à régulariser une situation, sans volonté réformatrice dans le secteur de l'entretien, marque peut-être la fin de la transition dans le secteur du logement : dans

51. Entretien avec I. Simanoviča, directrice du département du Logement de l'agence de l'État pour la Construction, les performances énergétiques et le logement, 16 avril 2009.

52. Entretien avec J. Baiks, secrétaire général de la ville de Valmiera, 6 avril 2009. Entretien avec D. Ošenieks, 20 avril 2009. 
la mesure où il désigne les offices publics du logement comme gestionnaires en titre d'un grand nombre de copropriétés privées, il qualifie de pérenne une situation décrite jusqu'alors comme transitoire ${ }^{53}$.

\section{RÉFÉRENCES BIBLIOGRAPHIQUES}

Amestoy I. (2001), «La privatisation des logements urbains : un processus complexe et en évolution. Le cas de Saint-Pétersbourg », Revue d'études comparatives Est-Ouest, vol. 32, n 4, pp. 153-183.

ANDRIA A. (2007), Rapport sur la politique du logement et la politique régionale, Commission du développement régional du Parlement européen, 28 mars, 15 pages.

BEMA (2009), Pārskats par dzīvojamo māju privatizācijas norisi 2008. gadā (Compte rendu du déroulement de la privatisation durant l'année 2008), Riga ; http://polsis.mk.gov.lv

Bourdieu P. (1993), «Effets de lieu », in P. Bourdieu, La misère du monde, Paris : Seuil, pp. 159-167.

ConseIl des ministres (1996), Mājoklu politikas koncepcija (Conception de la politique du logement), Riga, 30.07. Disponible sur le site du ministère letton de l'Économie : www.em.gov.lv

Czischke D. \& PitTini A. (2007), Housing Europe 2007. Rapport sur le logement social, le logement public et le logement en coopérative dans les 27 États membres de l'UE, Bruxelles : Observatoire du logement social européen du CECOHDAS, octobre.

DesjaRdins X. (2008), «Le logement social au temps du néolibéralisme », Métropoles, 18.12.; http://metropoles.revues.org

Eesti statistika (2009) (Agence de Statistiques du ministère estonien des Finances).

Eurostat (2010), « Conditions de vie en 2008 », Service de presse d'Eurostat, 18.01.; epp.eurostat.ec.europa.eu

E\&IC (2006), Labās prakses noteikšana un izvērtēšana pašvaldības iespējām revitalizêt dzīivojamos rajonus (Évaluation et détermination pour la municipamité des principes de bonne pratique dans la revitalisation des quartiers résidentiels), Riga ; www.rdpad.lv

53. Entretien avec G. Ābele, directeur, et M. Auders, chef du bureau de la politique du logement, ministère de l'Économie de Lettonie, 15 juillet 2009.

VOLUME 41, JUIN 2010 
Foucault M. (2004), Naissance de la biopolitique. Cours au Collège de France. 1978-1979, Paris : Seuil.

GHÉKIÈRe L. (2007a), Le développement du logement social dans l'Union européenne. Quand l'intérêt général rencontre l'intérêt communautaire, Paris : Dexia.

GHÉKİ̀re L. (2007b), «Le logement social dans l'Union européenne : nouvelle donne à 27 États », Habitat et Société, n 46, pp. 26-56.

KAHN M. (1992), «Le logement dans l'ex-URSS », Le Courrier des pays de $l^{\prime} E s t, \mathrm{n}^{\circ}$ 371, La Documentation française, pp. 54-67.

Kronberga G. (2005), "Vai ḷaus pārdot neprivatizētos dzīvokḷus ?" (Pourra-ton vendre les appartements qui n'ont pas été privatisés ?), Latvijas Vēstnesis, 02.09 ; www.vestnesis.lv

Leckie S., Pomykala A. \& Scherer A. (2000), Housing Rights in Latvia, Centre on Housing Rights and Evictions ; www. cohre.org

Loseva I. \& Zvejnieks A. (2004), "Mājokḷu situācijas analīze Latvijā" (Analyse de la situation du logement en Lettonie), Actes de la conférence Tautsaimniecības un uznềmējdarbības attīstības problēmas (Les problèmes de l'économie nationale et de l'entrepreneuriat), Riga : Université technique de Riga, 9-11 octobre 2003.

Marana I. \& ZaḲIs V. (2004), "Social Housing in Latvia: Current Situation and the Future Perspective", in Social Housing, UNECE Conference, Vienna, 28-30 November 2003 ; www.europaforum.or.at

Ministère de la Protection de l'enVironnement et du déVeloppement RÉGIONAL (2002), Mājokḷu politika Latvijā (La politique du logement en Lettonie), Riga, 12 pages.

Ruoppila S. (2004), "Processes of Residential Differentiation in Socialist Cities: Literature Review on the cases of Budapest, Prague, Tallinn and Warsaw", European Journal of Spatial Development, $\mathrm{n}^{\circ}$ 9, February ; www.nordregio. se/EJSD/

ŠČUCKA S. (2004), “Īres griestu atcelšanas cilvēktiesību aspekti” (Suppression des plafonds de loyer et droits de l'homme) », Politika, 07.09.; www.politika. lv

S.I.A. Tursons (2004), Rīgas iedzīvotāju mājokḷu apstākḷu, kā arī mājokḷu pieejamības raksturojums un priekšlikums par minimālo mājokḷa standartu 2018. gadam (Caractérisation des conditions de logement et de l'accès au logement des habitants de Riga et proposition d'un standard minimal de l'habitat pour l'année 2018), Riga ; www.rdpad.1v

SmițGe S. (2008), "Pretējā virzienā" (À contre-courant), Sestdiena, 16.08., pp. 16-17.

Statistikos Departamentas prie Lietuvos Respublikos Vyriausybès (Département des statistiques du gouvernement de la République de Lituanie); www.stat.gov.lt 
194 ERIC Le Bourhis

Tsenkova S. \& Turner B. (2004), "The future of social housing in Eastern Europe: Reforms in Latvia and Ukraine", International Journal of Housing Policy, Vol. 4, n 2, August, pp. 133-149. Texte présenté lors de la conférence Adequate and Affordable Housing for All, Toronto: University of Toronto, June.

VAsILJEvs V.(2005),"Privatizācijas sertifikātu pirmsākums Latvijā.Privatizācijas sertifikāts - no ārzemēm importēts produkts" (L'origine des certificats de privatisation en Lettonie : le certificat de privatisation - un produit importé de l'étranger), Latvijas Véstnesis, 08.04.; www.vestnesis.lv 